Oxide of methyle $\mathrm{C}_{2} \mathrm{H}_{3} \mathrm{O}$

Chloroform ....... $\mathrm{C}_{2} \mathrm{H} \mathrm{Cl}_{2} \mathrm{Cl}=\mathrm{C}_{2} \mathrm{H} \mathrm{Cl}_{2}$.

Its relation to formic acid is represented by the replacement of three atoms oxygen by three atoms chlorine, as follows:-

$$
\begin{aligned}
& \mathrm{C}_{2} \\
& \mathrm{C}_{2}
\end{aligned} \mathrm{H} \mathrm{O}_{3}=\text { Formic acid. }
$$

The following table exhibits a few trials which $\mathrm{I}$ made of the preparation of chloroform by distillation, with various properties of alcohol, pyroxilic spirit, and brewer's wash. The experiments were conducted in the college laboratory, under the superintendence, and at the request, of Dr. R. D. Thomsom.

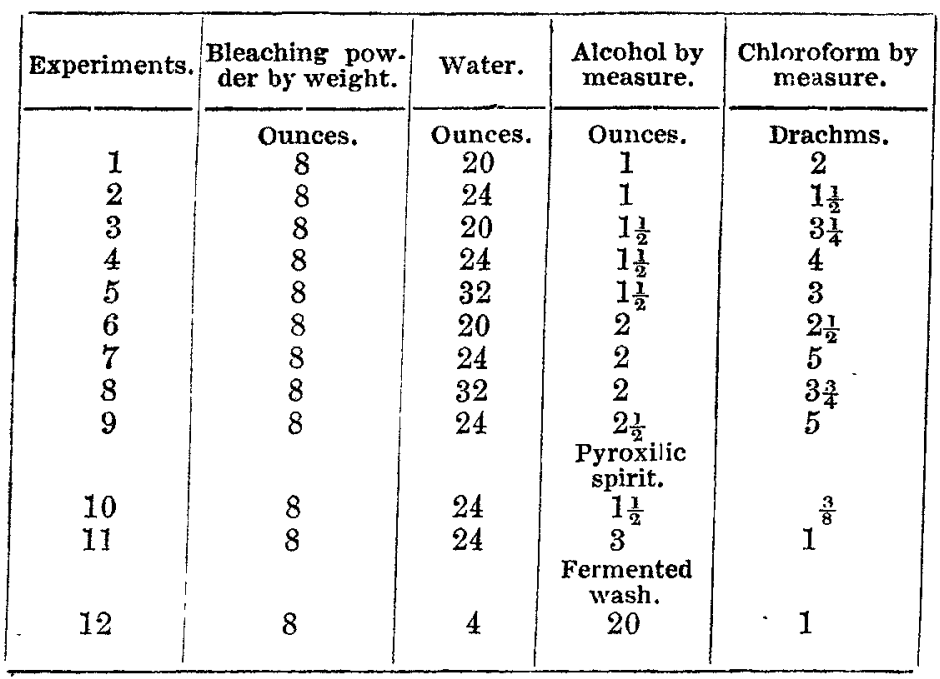

The specific gravity of the alcohol was 0.837 ; the specific gravity of the pyroxilic spirit, 0.838 .

The substances were first mixed in a glass vessel, and then introduced into a retort of the capacity of half a gallon.

The following specimens were exhibited to the Society:

In No. 1 is chloroform which has not been washed, but in the condition in which it was distilled; its specific gravity is 1.226 .

In No. 2 is chloroform which has been washed with distilled water until the water with which it was washed gave no precipitate with nitrate of silver. Its specific gravity is 1.446 .

In No. 3 is chloroform which has been washed with distilled water agitated with chloride of calcium, and distilled with sulphuric acid; its specific gravity is 1.4995

I find it an advantage to remove the liquor which has been distilled with the chloroform as soon as possible; sixteen drachms of the distilled liquor generally absorbed half a drachm in twelve hours. If there has been a large amount of alcohol distilled with the chloroform, it absorbs more. From these trials the proportions of eight ounces of hypochlorite of lime, or bleaching powder, twenty-four ounces of water, and one ounce and a half of alcohol, give the best results. If one and a half give four, two ought to give five and one-third; it only gives five.

Chloroform is a colourless oily liquid, having an agreeable ethereal smell, and sweet taste. It is very slightly soluble in water, but soluble in alcohol and ether. It boils at a temperature of about $141^{\circ}$; its specific gravity is 1.4995 , or 1.5 .

There are several substances which, when inhaled into the lungs, cause stupor or insensibility. We have nitrous oxide, (the stupefacient effect of which gas was discovered by Sir H. Davy,) composed of one atom of nitrogen and one of oxygen. "We have sulphuric ether, composed of four atoms of carbon, five of hydrogen, and one of oxygen.

In October last, Dr. Simpson applied to Mr. Waldie of Liverpool, when in Edinburgh, to recommend an agent that possessed the properties of sulphuric ether. Mr. Waldie advised the use of chloroform, which Dr. Simpson tried, and found to be successful. At a meeting of the French Academy, held on the 29 th of November, 1847 , it was stated, that at the time the stupefacient effect of ether was observed, several attempts were made to find some other agent capable of producing the same effect; that at that time $M$. Flourens, se. cretary to the Society, having made some trials on animals, found that chloroform possessed the same power of rendering them insensible. Chloroform is supposed to act on the system in the same way as sulphuric ether. For an account of the action of sulphuric ether, I refer to a paper read by Dr. Andrew Buchanan, at a meeting of this Society on the 22nd of February, 1847 .
Dr. Simpson says the superiority of chloroform over sulphuric ether consists in its requiring a less quantity to produce the same effect, its action being much more rapid and complete, its inhalation being much more agreeable, its perfume not being unpleasant, and its odour not remaining attached to the clothes.

\section{CASE OF ENDOCARJITIS COUPLED WITH UNUSUAL ENLARGEMENT OF THE LIVER AND SPLEEN.}

Bx J. L. ION, Esq., M.R.C.S., \&c., London.

The patient, a female, aged twenty-seven years, had been seized eight years prior to her decease with occasional attacks of violent palpitation of the heart, accompanied by syncope and perspiration. These attacks continued withont much in. termission during the whole of the above-named period. The pulse at these times being remarkably deficient in force, during several of the catamenial visitations, she complained of intense pain and tenderness in the region of the spleen; this was well marked by her medical attendant during several successive periods. She had little or no cough; indeed, fainting and palpitation were the only symptoms that seemed to distress her. There was no deficiency in the assimilating organs, and she continued fleshy to the time of her decease. A peculiar bellows somnd was heard during life, when the left ventricle contracted, which was to be accounted for by the peculiar contracted condition of the edges of the mitral valve.

A post-mortem investigation revealed the following morbid appearances:-No emaciation; the cellular tissue throughout being well filled with adipose secretion. On opening the thoracic cavity, both sides were found nearly filled with a serosanguineous fluid, and the bag of the pericardium was distended with a similar production. The pleural and costal investitures were remarkably free from any signs of inflammatory action; indeed, it is seldom that this membrane is found more healthy than in this particular case. On dividing the pericardium throughout its entire length, the heart was discovered full one-third larger than its usual size, and its texture was denser and firmer than usual. The left ventricle was hypertrophied with dilatation of the walls. The carneæ columne were unnaturally large and firm, and there were general induration and thickening of the lining membrane of the cavities; large flakes of coagulable lymph could be disengaged from the endocardium, though the membrane beneath appeared healthy. But about the auriculo-ventricular valves, the most manifest alteration of structure presented itself: here the serous lining was much thickened and indurated, and large beads of opaque coagulable deposit formed a fringe upon the free edges of the valves, appearing like the white fringe upon window curtains. The ends of the polypi in the auriculoventricular opening were covered with lymphatic deposit, and the diseased state of the edges of the mitral valve in the left ventricle, no doubt, gave rise to the bellows sound at the time of its contraction. The semilunar valves were healthy, nor could any traces of diseased action be discovered in the muscular structure of the heart. The liver was enormously enlarged, particularly at its superior margin, but its internal structure was healthy. The spleen was of nearly three times its natural size, and much cangested throughout; on freely dividing it, no organic lesion was discoverable. The kidneys were much developed, but, independent of simple enlargement, were perfectly free from disease; indeed, the whole of the other viscera, with the exceptions above noticed, were remarkably healthy.

From these morbid appearances, two or three deductions may naturally be drawn. The hypertrophied-state of the three important organs-viz., heart, liver, and spleen-subsisting in the same subject, and at the same time, would naturally give rise to the inference, that some relative influence had been the cause of such a simultaneous enlargement. Physiology and pathology do not yet enable us to say in what precise relationship they stand to each other. My own opinion favours the supposition that the diseased condition of the great organ of circulation originated the other disorders, fa vouring irregular distribution of the blood. The enlargement of the abdominal organs had little to do in the fatal termination. Serous effusion, of a passive character, seems to have been the proximate cause of death; and why the liver and spleen should have participated in the enlargement, I am at a loss to conjecture.

This was evidently a case of pure endocarditis, and probably one of a chronic character. It is, I believe, a disease 
of more frequent occurrence than is generally supposed, especially in females. For a very able exposition of its symptoms, morbid anatomy, treatment, \&c., I beg to refer the profession to a very lucid paper, by Dr. Munk, in Tue Lancer of Sept. 19th, 1846.

I am permitted to give the foregoing details through the kindness of my friend Mr. Berrell, under whose care the patient remained to the time of her death, and who favoured me with his permission to be present at the post-mortem examination.

Moore-place, Kennington-road, 1849.

\section{Rebíctus.}

Lectures on the Causes and Treatment of Ulcers of the Lower Extremity. Delivered at the London Hospital during the Summer of 1848. By Georae Critchett, F.R.C.S., \&c. London: Churchill. 8vo. pp. 121.

A Treatise on the Cure of Ulcers by Fumigation. By G. 0 . WALKER, Surgeon, \&c. London, 1848. 8vo, pp. 112.

On the Treatment of Ulcers on the Leg without Confinement; with an Inquiry into the best mode of effecting the permanent cure of Varicose Veins. By Henry T. Chapman, F.R.C.S. London, 1848. 8vo, pp. 156.

The lectures of Mr. Critchett first appeared in The Lancet during the latter half of last year, and since they have been published in their present form we havealready briefly drawn attention to them, as containing important matter. Not that Mr. Critchett has announced any new plan of treatment in them which waits to receive the support of adequate experience; he has, on the contrary, repudiated this in his Preface, in which he remarks-

"There exists such a plethora in medical literature, that it behoves each new commentator upon Nature's morbid operations, if he would expect to obtain any attention from his professional brethren, to accomplish one of three objects; -he must either propound new and original views, founded on legitimate induction, or he must collect the rays of light that lie scattered over the fields of pathology, and present them in a clear, concentrated, and useful form; or, lastly, he must resuscitate some old principle, valuable in a practical point of view, which, from some accidental cause, has been allowed to slumber in neglect and oblivion; it is in this latter and humbler capacity that the author of these lectures breaks silence; he claims not the high honour of having discovered a new window, whereby light may be admitted into our surgical temple, but simply to have rendered transparent one that had become obscured and useless. The author's main object, then, in the publication of this little book, is to place the principle of mechanical support, in the treatment of ulcers of the lower limbs, on a correct and scientific basis; for although, as he has elsewhere endeavoured to show, this has already been briefly done by the late Mr. John Scott, in his work ' On Diseased Joints,' a reference to all the best surgical authorities since that work appeared, proves how eutirely that principle is still, if not unknown, at least most imperfectly expounded."

In the treatment of old ulcers of the lower extremity, Mr. Critchett depends much upon mechanical support, not accord. ing to Baynton's method, but after that adopted by the late Mr. Scott, of Bromley, his advocacy of which plan forms a main feature in his work. He gives practical instructions for the application of pressure, which have been already pub. lished, and illustrated with plates, in The Lancet, and to which we refer at pp. 25-30 of his work. On the applicability of the water-dressing to subacute ulcers, Mr. Critchett says:-

"Much has been said and written within the last few years on the subject of the 'water-dressing' for wounds. I have tried it extensively; it has the advantages of cleanliness, economy, and simplicity, and is certainly an immense improvement upon the complicated unguent of former days; but it is desirable, if possible, to get at its real value. It answers remarkably well in the transition stage from the subacute to the chronic ulcer; but there comes a time when the surface of the sore is either raised or flabby, as in cases where it is combined with entire rest, or becomes dry, and makes no progress towards healing. In either case it has done its work, and some other application must be substituted."
Chronic, healthy, irritable, varicose, strumous, phagedænic; menstrual, malignant, and the other varieties of ulcers are concisely treated of, and several illustrative cases have been introduced into the work. Our space is too limited at present to admit of our making further extracts, and we must sum up our remarks by expressing an opinion that Mr. Critchett's work, for its intrinsic merit, ought to find a place in the library of every surgeon.

Of no disease does a medical practitioner undertake the management more hopeless of permanent benefit, than $a$. "bad leg." Viewed in any light, the malady is associated, in his mind, with tediousness, perpetual trouble, and, in the majority of cases, with unsatisfactory results. This is evidently the consequence of unsettled methods of treatment. The hap-hazard system of practice has long had full play in the management of these diseases; for every practitioner has his own mode of treatment, and every nostrum has its day of popularity, only to be thrown aside and replaced by another, the fame of which becomes equally ephemeral. It is not, therefore, surprising that the quacks enjoy so large a share of this kind of practice. John Bull might well say, "it is impossible to be serious while we enumerate the thousand remedies which have been recommended for ulcers."

The monographs, the titles of which head this article, will serve to illustrate, and also in great measure to corrobowate, our remarks. They are put forth with the same object, that. of curing ulcers of the leg, but after totally different fashions, and on very different principles. Mr. Walker's volume contains the history of his own experience, for several years past, of a particular remedy, which he considers himself justified in recommending to the profession as superior to all others for the cure of ulcers.

It is composed of sulphur and iodine, in the form of vapour, the strength of which is to be varied according to the duration of the disease, and the amount of stimulus required. The author says,-

"He has repeatedly seen some of the most obstinate cases of habitual ulcer,-cases which had gone the round of the hospitals, and baffled the skill, while they exhausted the patience, of the surgeons,--yield, in a surprisingly short period of time, to the influence of the iodine and sulphur fumigation. This vapour seems to exert an influence at once profound and rapid on the organic nutrition of the parts. One of its first and most visible effects is to check the secretion of abnormal deposits from the inflamed capillaries, which are soon restored to their natural calibre, and to alter the morbil disposition which gives rise to ulcerative alssorption. It stimulates the vital energy of the cells of the cellular tissue, and enables their bloodvessels to secrete an increased quantity of nutritive matter from the fluids which they convey, thus affording a considerable amount of new and healthy tissue in a short space of time."

Mr. Walker is in error in supposing that Galés, in 1816 , was the first to apply sulphur fumigations to the skin. It was employed long before, at the Parisian Hospital of St. Louis, by Alibert.

The method of applying the vaponr to the diseased surface is thus described, and is a fair specimen of the author's style:-

"The apparatus is extremely simple. It is composed of a series of mahogany boxes, having in the cover of each a round aperture for the reception of the limb. Each box communicates below with the engine-room of my establishment, whence heated air or warm vapour can be passed into the apparatus. On the bottom of the box is placed a heated iron, and on the latter the powder of iodine and sulphur, which is volatilized by heat. The relative quantities of the medical agents-the time of application of the remedy-the temperature at which it is applied - the necessity of ealling to our aid heated dry air or vapour-these, and many other circumstances, must be regulated by the peculiarities of each individual case, and can scarcely be comprised in any general description. For ordinary purposes, a powder containing from two to three grains of iodine, to half a drachm of the flowers of sulphur, will suffice; but the relative proportions of the remedies must be varied according to the expediency of 